\title{
Longitudinal validation of periarticular bone area and $3 D$ shape as biomarkers for knee $O A$ progression? Data from the FNIH OA Biomarkers Consortium
}

\author{
David Hunter, ${ }^{1}$ Michael Nevitt, ${ }^{2}$ John Lynch, ${ }^{2}$ Virginia Byers Kraus, ${ }^{3}$ Jeffrey N Katz, ${ }^{4}$ \\ Jamie E Collins, ${ }^{4}$ Mike Bowes, ${ }^{5}$ Ali Guermazi, ${ }^{6}$ Frank W Roemer, ${ }^{6,7}$ Elena Losina, ${ }^{4}$ \\ for the FNIH OA Biomarkers Consortium
}

\begin{abstract}
Handling editor Tore K Kvien
- Additional material is published online only. To view please visit the journal online (http://dx.doi.org/10.1136/ annrheumdis-2015-207602)

For numbered affiliations see end of article.
\end{abstract}

\section{Correspondence to}

Dr Hunter at Rheumatology Department, Royal North Shore Hospital and Institute of Bone and Joint Research, Kolling Institute, University of Sydney, Sydney, NSW 2065, Australia; David.Hunter@sydney.edu.au

Received 15 March 2015 Revised 17 August 2015 Accepted 20 September 2015

Published Online First

19 October 2015

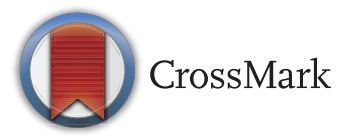

To cite: Hunter $\mathrm{D}$ Nevitt M, Lynch J, et al. Ann Rheum Dis 2016;75: 1607-1614.
ABSTRACT

Objective To perform a longitudinal validation study of imaging bone biomarkers of knee osteoarthritis (OA) progression.

Methods We undertook a nested case-control study within the Osteoarthritis Initiative in knees (one knee per subject) with a Kellgren and Lawrence grade of 1-3. Cases were defined as knees having the combination of medial tibiofemoral radiographic progression and pain progression at the 24-month, 36-month or 48-month follow-up compared with baseline. Controls $(n=406)$ were eligible knees that did not meet both endpoint criteria and included 200 with neither radiographic nor pain progression, 103 with radiographic progression only and 103 with pain progression only. Bone surfaces in medial and lateral femur, tibia and patella compartments were segmented from MR images using active appearance models. Independent variables of primary interest included change from baseline to 24 months in (1) total area of bone and (2) position on threedimensional (3D) bone shape vectors that discriminate $\mathrm{OA}$ versus non-OA shapes. We assessed the association of bone markers changes over 24 months with progression using logistic regression.

Results 24-month changes in bone area and shape in all compartments were greater in cases than controls, with ORs of being a case per 1 SD increase in bone area ranging from 1.28 to 1.71 across compartments, and per 1 SD greater change in 3D shape vectors ranging from 1.22 to 1.64. Bone markers were associated most strongly with radiographic progression and only weakly with pain progression.

Conclusions In knees with mild-to-moderate radiographic $\mathrm{OA}$, changes in bone area and shape over 24 months are associated with the combination of radiographic and pain progression over 48 months. This finding of association with longer term clinical outcome underscores their potential for being an efficacy of intervention biomarker in clinical trials.

\section{INTRODUCTION}

Osteoarthritis (OA) is a highly prevalent, disabling disease, with a commensurate tremendous individual and societal burden. ${ }^{1}$ Recent estimates suggest that 250 million people worldwide are affected by knee OA. ${ }^{2}$ Losina et $a l^{3}$ recently reported that individuals aged 50-84 years with knee OA experienced losses in quality-adjusted life-years (QALYs) over the remainder of their lives ranging from a mean of 1.9 QALYs in non-obese persons with knee OA to 3.5 QALYs for persons with knee OA who are obese. In the USA alone, 15 million QALYs are lost due to OA. ${ }^{3}$

There is a pressing need for biomarkers that serve to identify patients likely to progress radiographically, symptomatically or both, who can be targeted for therapy, and for biomarkers that reflect response to therapy in clinical trials. One potential tissue biomarker is subchondral bone; it is pivotally involved in the pathogenesis of $\mathrm{OA}$, is the tissue principally responsible for load distribution in the weight-bearing knee and is highly responsive to changes in load distribution. Bone is a promising area for OA biomarker qualification due to the multiple methods available to image periarticular bone, the variety of bone changes that are associated with development of $\mathrm{OA}^{4}$ and the abundant associations of bone biochemical markers with various aspects of OA. ${ }^{5}$ The changes in subchondral bone occurring in established knee OA include remodelling of the subchondral trabeculae, ${ }^{6-8}$ alterations in shape, ${ }^{9} 10$ thickening of the subchondral plate $^{11}$ and a steep stiffness gradient. ${ }^{12}$ Expansion of the subchondral surface area is well described in late stages of $\mathrm{OA}^{13}$ and may also be associated with early stages of knee OA development. ${ }^{14}$ In addition, subtle alterations in joint shape at both the hip and knee may be involved in the pathogenesis of OA. ${ }^{9}{ }^{15}$ A recent study found that differences in three-dimensional (3D) shape of the periarticular tibia and femur are associated with the subsequent onset of incident radiographic knee OA. ${ }^{16}$ Previous studies have suggested that bone area can predict development of cartilage defects, ${ }^{17}$ is larger in knees with radiographic $\mathrm{OA}^{14}$ and can predict the need for knee joint replacement. ${ }^{17}$ Whether changes over time in bone area or bone shape are associated with clinically relevant progression (not defined using joint replacement as the definition) of knee OA is at present unknown.

Under the auspices of the Foundation for the National Institutes of Health OA Biomarkers Consortium, we undertook a nested case-control 
study within the Osteoarthritis Initiative (OAI) to evaluate the longitudinal validity of a broad spectrum of imaging and biochemical markers of disease progression in knee OA. ${ }^{18}$ Here, we report the results for biomarkers of knee subchondral bone surface area and 3D bone shape derived from MR images. The objective of this study is to determine whether changes over 24 months in (1) subchondral bone surface area and (2) summary measures of overall shape for each knee bone are associated with radiographic and pain progression over 48 months in knees with mild-to-moderate OA.

\section{MATERIALS AND METHODS \\ Study design}

We used public data and images from the OAI, an ongoing multicentre observational cohort study of knee and hip OA (http:// www.oai.ucsf.edu/) designed to identify and qualify imaging, biochemical and genetic biomarkers for the onset and/or progression of knee OA, and funded by the NIH and industry. ${ }^{19}$ The OAI enrolled 4796 subjects ages $45-79$ at four clinical centres. Participants had, or were at increased risk for, symptomatic knee $\mathrm{OA}$ in at least one knee at enrolment and have been followed for up to 8 years. ${ }^{19}{ }^{20}$ Imaging (MRI and radiography) of both knees of participants, serum and urine specimens and clinical data were obtained at baseline, 12, 24, 36 and 48 months of follow-up.

Eligible participants were those with at least one knee with a Kellgren and Lawrence grade (KLG) (27) of 1, 2 or 3 at baseline from central reading ${ }^{21}$ (to exclude persons who definitely did not have OA (KLG0) but were not end stage (KLG4)) and availability at baseline and 24 months of knee radiographs, knee MRI, stored serum and urine specimens and clinical data. We excluded participants with a total knee or hip replacement from baseline through 24 months due to potential effects on biochemical markers.

\section{Radiographic progression}

Radiography of both knees was performed at all clinic visits using the same non-fluoroscopic fixed flexion protocol (SynaFlexor, Synarc, Newark, California, USA). ${ }^{22}$ Radiographs were assessed by central reading ${ }^{23} 24$ for $\mathrm{KLG}^{25}$ and semiquantitative joint space narrowing (JSN) using the Osteoarthritis Research Society International atlas. ${ }^{26}$ The minimum joint space width (minJSW) in the medial femorotibial compartment was measured using automated software. ${ }^{27}$ Radiographic progression was defined as a decrease from baseline to 24,36 or 48 months in minJSW of $\geq 0.7 \mathrm{~mm}$. This cut-off was selected based on the mean and SD of 1-year changes in minJSW in 90 OAI reference control group knees with a KLG of 0 and the Western Ontario and McMaster Universities Arthritis Index (WOMAC) pain scores of 0 at both timepoints; a decrease of $\geq 0.7 \mathrm{~mm}$ has a $10 \%$ probability of being due to measurement error and is consistent with values for the minimum detectable differences in medial minJSW using other methods. ${ }^{21}$ In the OAI, the intraclass correlation for a repeat measurement by the same observer for the change in minJSW over 36 months in OAI knees is 0.96 .

We excluded knees with poor positioning on knee radiographs (defined in terms of tibial plateau rim distance) that would make measurement of medial compartment JSW unreliable and knees that were unable to meet criteria for progression due to ceiling effects at baseline. We also excluded knees with predominantly lateral compartment JSN at baseline or during follow-up, based on semi-quantitative assessments, ${ }^{28}$ to avoid misclassification on radiographic progression based on JSW changes in the medial compartment only.

\section{Pain progression}

Knee pain was assessed using the WOMAC pain subscale. ${ }^{29}$ Pain progression was defined as a persistent increase from baseline to 24,36 or 48 months of $\geq 9$ points on a $0-100$ normalised score, based on the literature for a minimum clinically important difference for pain worsening. ${ }^{30} 31$ Persistence required a pain increase of $\geq 9$ points at $\geq 2$ timepoints from the 24 -month to 60-month pain assessment. Knees were also excluded if there were not enough follow-up timepoints after the first increase in WOMAC pain data above the threshold to determine whether the increase was persistent.

\section{Study knees and subjects}

For the nested case-control study, we selected a predetermined number of index knees, one knee per participant, in four different knee outcome groups: (1) knees with both radiographic and pain progression - these were the primary case knees; (2) knees with radiographic but not pain progression; (3) knees with pain but not radiographic progression; and (4) knees with neither radiographic nor pain progression. If both of a participant's knees fell into any one group, one was randomly selected as the index knee. Imaging biomarkers were assessed in the index knee.

Since the primary independent variables were biomarker changes during the first 24 months of follow-up and outcomes were defined by changes occurring at 24 months and after, we did not include participants with a knee that already had radiographic and pain progression at 12 months. In addition, since systemic (serum and urine) biochemical markers are a personlevel variable, we also made the following exclusions based on contralateral knee: the contralateral knees of participants in group 2 could not have pain progression and in group 3 could not have radiographic progression. Those with an index knee selected for group 4 could not have radiographic progression, including worsening of lateral compartment JSN or pain progression in the contralateral knee.

The prespecified sample size goal for the four groups was 200, 100, 100 and 200 knees, respectively. For better covariate balance among the groups, and to the extent feasible, knees selected for the four groups were frequency matched for KLG and body mass index $(\mathrm{BMI})\left(\mathrm{kg} / \mathrm{m}^{2}\right)$ categories $(<25 ; 25$ to $<27.5 ; 27.5$ to $<30 ; 30$ to $<35$; $\geq 35$ ). MRIs of the selected index knees were reviewed for artefacts that would interfere with image analysis, and if present, this knee and subject were excluded and a replacement selected (see figure 1 for subject flow diagram).

\section{MRI sequence parameters}

MRI acquisition was performed using a 3 T MRI system (Trio, Siemens Healthcare, Erlangen, Germany) at the four OAI clinical sites. The MRI pulse sequence protocol included a coronal two-dimensional (2D) intermediate-weighted (IW) turbo spin-echo, sagittal 3D dual-echo at steady-state (DESS), coronal and axial multiplanar reformations of the 3D DESS and sagittal IW fat-saturated turbo spin echo (TSE) sequences. Additional parameters of the full OAI pulse sequence protocol and sequence parameters have been published in detail. ${ }^{20}$ The current analysis used the double-echo-in-steady-state sequence (DESS-we) of the Siemens $3 \mathrm{~T}$ trio systems.

\section{MRI image processing}

Femur, tibia and patella bone surfaces were automatically segmented from $3 \mathrm{~T}$ DESS-we images using active appearance 
$\mathrm{N}$ of subjects ( $\mathrm{N}$ of knees)

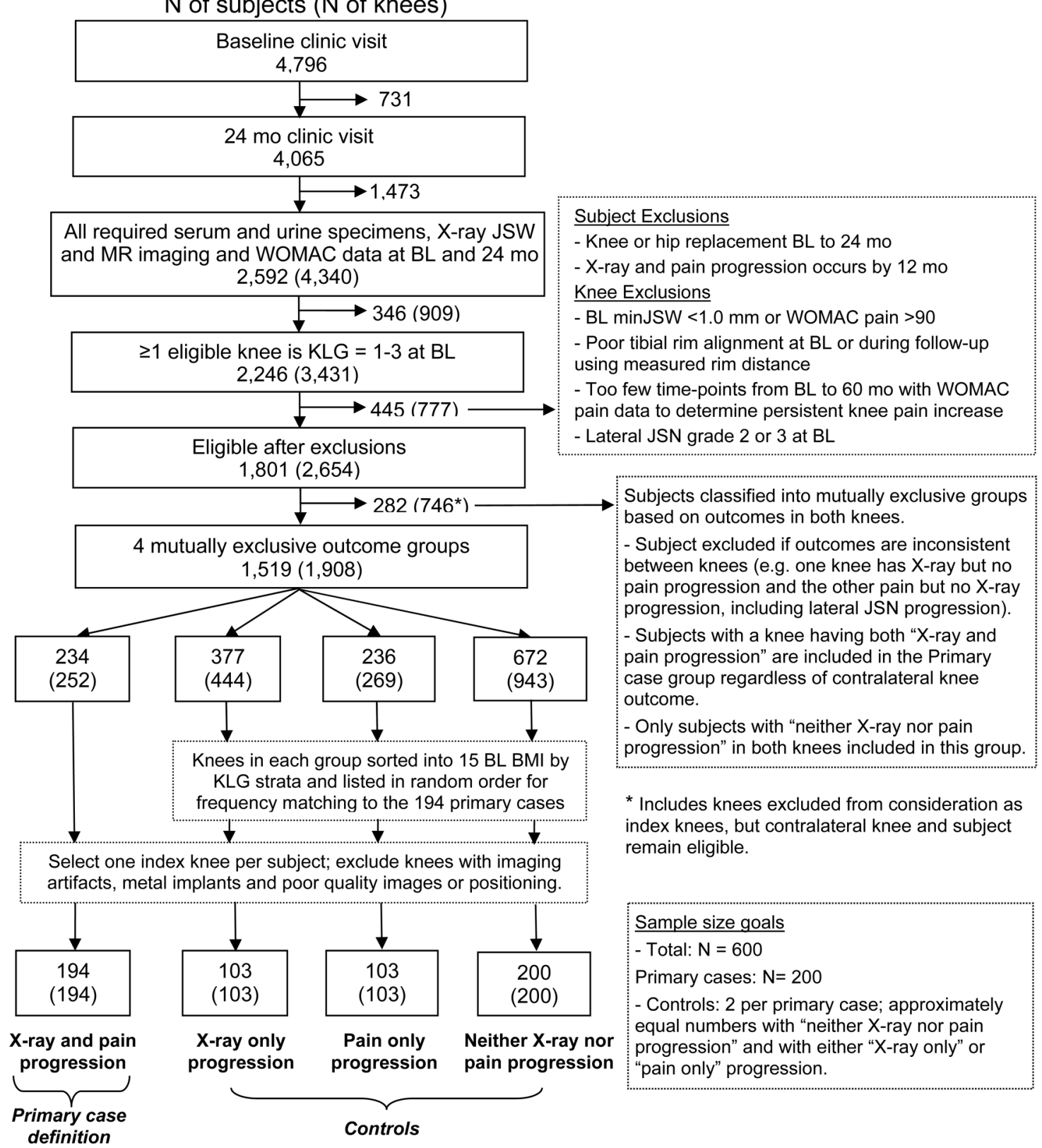

Figure 1 Participant flow diagram showing the selection of subjects for the study. BL, baseline; BMI, body mass index; JSN, joint space narrowing; JSW, joint space width; KLG, Kellgren and Lawrence grade; WOMAC, the Western Ontario and McMaster Universities Arthritis Index.

models (AAMs) provided by Imorphics (Manchester, UK) in a multistage process. First, a training set of 96 knee MRIs, using the DESS-we sequence, was used to build AAMs for the tibia and femur. ${ }^{32}$ This training set was selected to contain examples of each stage of OA with knees fulfilling each KLG (43 KLG0 and KLG1, 7 KLG2, 28 KLG3, 18 KLG 4). Anatomical regions were identified on the mean bone shape as described previously (figure 2A). ${ }^{33}$ We used a definition of the area of subchondral bone or 'tAB' similar to that designated by a nomenclature committee. ${ }^{34}$ However, this definition was modified to include bone ('peripheral osteophytes') from around the cartilage plate. The boundary between the medial femur (MF)/ medial trochlear femur and the lateral femur/lateral trochlear femur boundary in the femur was defined as a line on the bone corresponding to the anterior edge of the medial or lateral meniscus, and extended smoothly to the edge of the tAB (see online supplementary appendix video). The medial compartment of the tibiofemoral joint was selected to compare medial MF tAB and medial tibia (MT) tAB with medial joint radiographic measures on the basis that this compartment is more frequently affected.

Two measures of bone shape were used as primary independent variables: (1) the change in $\mathrm{tAB}$ area on the medial and lateral femur, tibia and patella, from baseline to 24 months; and (2) the change in position on $3 \mathrm{D}$ shape vectors for the femur, tibia and patella bones. The OA vector is the line passing through the mean shape for the two populations. The shape vector for each bone is calculated by taking the principal components of the mean non-OA shape, and the mean OA shape and drawing a straight line through them. The individual bone shape of study participants is represented by the same principal components as a consequence of using the AAM to search the images. Individual bone shapes are projected orthogonally onto the vector. Distances along the vector are normalised to a 
Figure 2 Anatomical regions and three-dimensional vector of shape change due to osteoarthritis (OA) in the femur. (A) The regions used in this study, displayed on the mean shape for each bone. The medial femur (MF)/ MedPF and the lateral femur (LF)/LatPF boundaries were defined as a line on the bone corresponding to the anterior edge of the medial or lateral meniscus in the mean model. The MedPF/LatPF boundary was defined as the centre of the trochlear groove in the mean model. (B) Shape examples along the femoral shape vector. The shape vector is calculated by taking the principal components of the mean non-OA shape, and of the mean OA shape, and drawing a straight line through them. Individual bone shape is represented using the same principal components and are projected onto the vector. Distances along the vector are normalised so that +1 represents the mean non-OA shape and -1 the mean OA shape. LatTr, lateral trochlear femur; LP, lateral patella; LT, lateral tibia; MedTr, medial trochlear femur; MP, medial patella; MT, medial tibia; PF, patellofemoral.

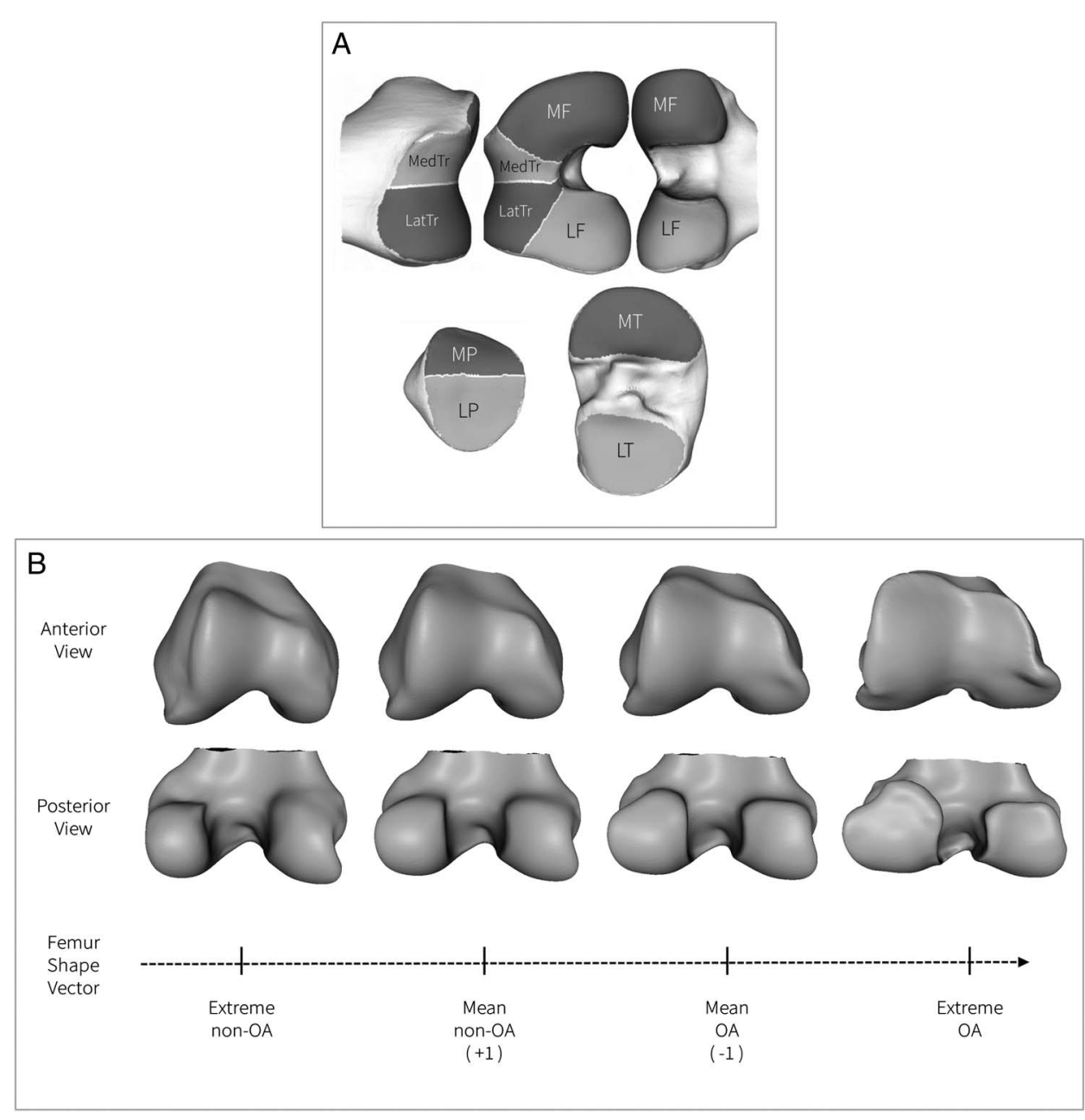

z-scale with the mean $\mathrm{OA}$ shape represented as -1 and the mean non-OA shape as +1 (figure $2 \mathrm{~B}$ ).

Reproducibility of the method was tested in a separate set of 35 OA knees that were imaged 1 week apart using the same OAI image acquisition sequence at a single OAI site. Reproducibility for the OA vector was good, with SD difference (SDD) (95\% confidence limits on a Bland-Altman plot) being $0.3(1 \%)$ normalised units for the whole joint model. ${ }^{33}$ These are presented in the online supplementary table S2.

\section{Statistical analysis}

We examined the association of change in bone morphometry biomarkers from baseline to 24 months with knee OA progression using logistic regression. First, in our primary analysis we compared change in bone biomarkers over 24 months in knees that had both radiographic and pain progression (group 1) with the other three groups of knees combined, that is, radiographic progression only (group 2), pain progression only (group 3) and knees with neither radiographic or pain progression (group 4). Next, we conducted a series of four secondary analyses. First, we used multinomial logistic regression with generalised logits to compare each group of progressor knees (groups 1-3) with knees that did not show any progression (group 4) (method 1). Second, we combined the three groups of progressor knees (groups 1-3) and compared this with non-progressor knees (group 4) (method 2). Third, we compared all knees that had radiographic progression (groups 1 and 2) with all knees that did not show radiographic progression (groups 3 and 4) (method 3). Finally, all knees with pain progression (groups 1 and 3) to all knees without pain progression (groups 2 and 4) (method 4).

Logistic regression models were adjusted for baseline values of minJSW, WOMAC pain, age, BMI, KLG, use of pain medications, as well as sex and race. Associations are expressed as the increase in odds of being a progressor knee for each 1 SDD in the change in a bone marker. To maintain overall type I error $<0.05$, the adjusted ORs were considered statistically significant if $\mathrm{p}<0.004$.

\section{RESULTS}

By design, the groups had similar age (mean 61.5 years, SD $8.9)$, sex distribution (59\% female) and the majority were obese (mean BMI $30.7 \mathrm{~kg} / \mathrm{m}^{2}$ ) (table 1). At baseline, the majority of knees were KLG 2-3 and JSW was $3.8 \mathrm{~mm}$. The two main comparison groups were similar in age, sex distribution and BMI.

\section{Radiographic and pain progressors versus all others}

Changes in bone area and shape from baseline to 24 months in all compartments were greater in radiographic and pain progressors (group 1) compared with knees that did not have both radiographic and pain progression (groups 2-4). The adjusted ORs in the table represent the change in odds of being a 'radiographic and pain progressor knee' versus a 'non-radiographic and pain progressor knee' per 1 SD increase of change in area and shape bone markers. Increases in bone area and the shape vector were associated with an increased odds of being a radiographic and pain progressor knee (table 2). The ORs per $1 \mathrm{SD}$ increase in change for bone area ranged from 1.28 to 1.71 
Table 1 Characteristics of study participants

\begin{tabular}{lll}
\hline & $\begin{array}{l}\text { Radiographic and } \\
\text { pain progressors } \\
\text { (group 1, N=194) }\end{array}$ & $\begin{array}{l}\text { Knees that did not have } \\
\text { both radiographic and } \\
\text { pain progression } \\
\text { (groups 2-4, N=406) }\end{array}$ \\
\hline Mean age (SD) & $62.0(8.8)$ & $61.3(8.9)$ \\
\hline Female sex (\%) & 56.7 & 59.9 \\
\hline BMI (kg/m²); mean (SD) & $30.7(4.8)$ & $30.7(4.8)$ \\
\hline History of injury to study knee & 35.2 & 35.8 \\
\hline -no (\%) & & \\
\hline KLG at baseline n, (\%) & & $51(12.6)$ \\
\hline 1 & $24(12.4)$ & $222(54.7)$ \\
\hline 2 & $84(43.3)$ & $133(32.8)$ \\
\hline 3 & $86(44.3)$ & $13.0(16.7)$ \\
\hline $\begin{array}{l}\text { Baseline WOMAC pain score; } \\
\text { mean (SD) }\end{array}$ & $10.2(13.0)$ & $3.9(1.1)$ \\
\hline Baseline JSW; mean (SD) & $3.8(1.4)$ & \\
\hline
\end{tabular}

BMI, body mass index; JSW, joint space width; KLG, Kellgren and Lawrence grade; WOMAC, the Western Ontario and McMaster Universities Arthritis Index.

across compartments, and for changes in shape ranged from 1.22 to 1.64 , with the largest ORs associated with bone changes in the MF and trochlea. Similar (although slightly weaker) effects are noted in the lateral tibiofemoral compartment and in the patella.

\section{Secondary comparisons of progressors versus non-progressors}

When comparing the radiographic and pain progressors (group 1) with those with no progression (group 4) on either factor, there was a slight increase in odds (table 3) compared with the data in table 2. The range of odds was from 1.38 to 2.69 for $1 \mathrm{SD}$ increase in bone area and 1.40-2.34 for changes in shape.

When comparing the odds of any progression $(n=400)$ with the non-progressor group $(n=200)$ (table 4, method 2), the ORs ranged from 1.25 to 2.13 for $1 \mathrm{SD}$ increase in area and 1.33 to 1.86 for shape. When comparing the odds of being a radiographic progressor $(n=297)$ with the radiographic nonprogressor group $(n=303)$ (table 4 , method 3$)$, the odds ranged from 1.29 to 2.62 for $1 \mathrm{SD}$ increase in area and 1.26 to 2.56 for shape. When comparing the odds of being a pain progressor $(\mathrm{n}=297)$ with the pain non-progressor group $(\mathrm{n}=303)$ (table 4 , method 4), the odds ranged from 1.17 to 1.30 for 1 SD increase in area and 1.16 to 1.23 for shape. In general, the odds were higher for identifying radiographic progressors (table 3, radiographic and pain progressor vs non-progressor, radiographic only progressor vs non-progressor; table 4, method 3) than for those distinguishing pain progressors from pain non-progressors (table 3, pain only progressor vs non-progressor; table 4, method 4).

We conducted additional exploratory analyses using baseline alone and/or changes from baseline to 12 months (as opposed to change from baseline to 24 months in bone area and shape) as the exposure variables. These are presented in the online supplementary table $\mathrm{S} 1$. The baseline to 12 -month findings are consistent in direction with those for baseline to 24 months.

\section{DISCUSSION}

Greater increases in bone area and shape markers over 24 months in knees with mild-to-moderate radiographic OA are associated with increased likelihood of clinically relevant progression consisting of a combination of radiographic and symptomatic progression over 48 months. In analyses examining the association of change in biomarkers with radiographic and pain progression as separate outcomes, we found a strong

Table 2 Change in bone area and shape for radiographic and pain progressors $(n=194)$ versus knees that did not have both radiographic and pain progression $(n=406)$

\begin{tabular}{|c|c|c|c|c|}
\hline & \multicolumn{2}{|l|}{ Means (SD) } & \multirow{2}{*}{\multicolumn{2}{|c|}{$\begin{array}{l}\text { OR }(95 \% \mathrm{Cl}) \text { for being a progressor per } \\
1 \mathrm{SD} \text { increase in change } \\
\text { p Value }\end{array}$}} \\
\hline & $\begin{array}{l}\text { Radiographic and pain } \\
\text { progressors (group 1, } \mathrm{N}=194 \text { ) }\end{array}$ & $\begin{array}{l}\text { Knees that did not have both } \\
\text { radiographic and pain progression } \\
\text { (groups } 2-4, N=406 \text { ) }\end{array}$ & & \\
\hline \multicolumn{5}{|l|}{ Medial area } \\
\hline Femur & $45.15(49.22)$ & 24.91 (36.49) & 1.71 (1.40 to 2.09 ) & $<0.0001$ \\
\hline Tibia & $20.02(22.42)$ & $13.07(20.20)$ & 1.42 (1.18 to 1.70$)$ & 0.0002 \\
\hline \multicolumn{5}{|l|}{ Lateral area } \\
\hline Femur & $13.42(38.17)$ & $5.94(33.04)$ & 1.28 (1.06 to 1.55$)$ & 0.0101 \\
\hline Tibia & $12.70(16.14)$ & 7.07 (13.86) & 1.50 (1.25 to 1.80$)$ & $<0.0001$ \\
\hline Patella & $13.10(20.45)$ & $6.05(20.65)$ & 1.45 (1.20 to 1.76$)$ & 0.0001 \\
\hline Notch & $16.69(25.07)$ & $10.06(22.53)$ & 1.29 (1.07 to 1.54$)$ & 0.0060 \\
\hline \multicolumn{5}{|l|}{ Lateral area } \\
\hline \multicolumn{5}{|c|}{ Shape (vector) } \\
\hline Femur & $0.30(0.34)$ & $0.17(0.27)$ & 1.64 (1.35 to 2.00$)$ & $<0.0001$ \\
\hline Tibia & $0.35(0.42)$ & $0.19(0.39)$ & 1.56 (1.29 to 1.88$)$ & 0.0003 \\
\hline Patella & $0.29(0.65)$ & $0.17(0.65)$ & 1.22 (1.01 to 1.46$)$ & 0.0348 \\
\hline
\end{tabular}

ORs $(95 \% \mathrm{Cl})$ and $\mathrm{p}$ values for being a progressor knee for a $1 \mathrm{SD}$ greater increase in bone markers, adjusted for baseline joint space width, baseline Western Ontario and McMaster Universities Arthritis Index pain, baseline age, baseline body mass index, baseline Kellgren and Lawrence grade (KLG), baseline pain medication use, sex and race. 
Table 3 Change in bone area and shape in progressor knees versus being in a reference group of knees having no radiographic and no pain progression (group 4, $\mathrm{n}=200$ ) (method 1)

\begin{tabular}{|c|c|c|c|}
\hline & Radiographic and pain progressor (group 1, $n=194$ ) & Pain only progressor (group $2, n=103$ ) & Radiographic only progressor (group $3, n=103$ ) \\
\hline \multicolumn{4}{|l|}{ Medial } \\
\hline Femur & 2.69 (2.03 to 3.56$) ;<0.0001$ & 1.13 (0.81 to 1.57$) ; 0.4682$ & 2.86 (2.09 to 3.92$) ;<0.0001$ \\
\hline Patella & 1.57 (1.25 to 1.97$) ;<0.0001$ & $1.12(0.86$ to 1.45$) ; 0.4132$ & 1.38 (1.06 to 1.80$) ; 0.0156$ \\
\hline \multicolumn{4}{|l|}{ Lateral } \\
\hline Femur & 1.38 (1.11 to 1.71$) ; 0.0043$ & $1.06(0.82$ to 1.37$) ; 0.6749$ & 1.21 (0.94 to 1.56$) ; 0.1421$ \\
\hline Patella & 1.67 (1.33 to 2.10$) ;<0.0001$ & 1.14 (0.87 to 1.48$) ; 0.3403$ & $1.45(1.10$ to 1.90$) ; 0.0076$ \\
\hline Notch & 1.59 (1.27 to 1.99$) ;<0.0001$ & $1.16(0.88$ to 1.51$) ; 0.2884$ & 1.78 (1.37 to 2.31$) ;<0.0001$ \\
\hline \multicolumn{4}{|l|}{ Lateral } \\
\hline Trochlea & 1.60 (1.29 to 2.00$) ;<0.0001$ & $0.98(0.75$ to 1.28$) ; 0.9034$ & 1.39 (1.07 to 1.79$) ; 0.0119$ \\
\hline \multicolumn{4}{|l|}{ Medial } \\
\hline Patella & 1.40 (1.12 to 1.75$) ; 0.0030$ & 1.27 (0.97 to 1.64$) ; 0.0777$ & 1.30 (1.01 to 1.68$) ; 0.0457$ \\
\hline
\end{tabular}

ORs $(95 \% \mathrm{Cl})$ and $\mathrm{p}$ values for being a progressor knee for a 1 SD greater increase in bone biomarkers, adjusted for baseline joint space width, baseline Western Ontario and McMaster Universities Arthritis Index pain, baseline age, baseline body mass index, baseline Kellgren and Lawrence grade (KLG), baseline pain medication use, sex and race.

relationship between these bone biomarkers and radiographic progression as the bone biomarkers are associated only weakly with progression in knee pain.

\begin{tabular}{|c|c|c|c|}
\hline & $\begin{array}{l}\text { Method } 2 \\
\text { Any progression } \\
\text { OR }(95 \% \mathrm{Cl}) \text { per } \\
1 \text { SD increase in } \\
\text { change }\end{array}$ & $\begin{array}{l}\text { Method } 3 \\
\text { Radiographic } \\
\text { progression } \\
\text { OR }(95 \% \mathrm{Cl}) \text { per } \\
1 \text { SD increase in } \\
\text { change }\end{array}$ & $\begin{array}{l}\text { Method } 4 \\
\text { Pain progression } \\
\text { OR }(95 \% \mathrm{Cl}) \text { per } \\
1 \text { SD increase in } \\
\text { change }\end{array}$ \\
\hline \multicolumn{4}{|l|}{ Medial } \\
\hline Femur & 2.13 (1.68 to 2.71$)$ & 2.62 (2.07 to 3.34 ) & $1.30(1.08$ to 1.56$)$ \\
\hline Tibia & 1.63 (1.33 to 1.99$)$ & 1.84 (1.51 to 2.24$)$ & 1.17 (0.99 to 1.39$)$ \\
\hline Patella & 1.39 (1.15 to 1.68$)$ & 1.45 (1.21 to 1.75$)$ & $1.25(1.05$ to 1.48$)$ \\
\hline \multicolumn{4}{|l|}{ Lateral } \\
\hline Femur & 1.25 (1.04 to 1.50$)$ & 1.29 (1.08 to 1.55$)$ & $1.17(0.98$ to 1.40$)$ \\
\hline Tibia & 1.51 (1.24 to 1.83 ) & $1.73(1.43$ to 2.10$)$ & $1.22(1.03$ to 1.45$)$ \\
\hline Patella & 1.45 (1.19 to 1.76$)$ & 1.52 (1.26 to 1.84$)$ & 1.29 (1.08 to 1.54$)$ \\
\hline Notch & 1.51 (1.24 to 1.83 ) & 1.57 (1.31 to 1.89$)$ & $1.16(0.98$ to 1.37$)$ \\
\hline \multicolumn{4}{|l|}{ Lateral } \\
\hline Trochlea & 1.35 (1.12 to 1.63$)$ & 1.53 (1.27 to 1.84$)$ & $1.21(1.02$ to 1.44$)$ \\
\hline \multicolumn{4}{|l|}{ Medial } \\
\hline Trochlea & 1.71 (1.38 to 2.12 ) & 2.02 (1.63 to 2.49 ) & $1.26(1.06$ to 1.50$)$ \\
\hline \multicolumn{4}{|c|}{ Shape (vector) } \\
\hline Femur & 1.86 (1.48 to 2.33 ) & 2.56 (2.03 to 3.24$)$ & $1.16(0.97$ to 1.39$)$ \\
\hline Tibia & 1.42 (1.18 to 1.72 ) & 1.83 (1.51 to 2.22$)$ & $1.16(0.98$ to 1.37$)$ \\
\hline Patella & 1.33 (1.10 to 1.62$)$ & 1.26 (1.05 to 1.50$)$ & $1.23(1.03$ to 1.47$)$ \\
\hline
\end{tabular}

Statistics presented in each cell: OR $(95 \% \mathrm{Cl})$. Adjusted for baseline joint space width, baseline Western Ontario and McMaster Universities Arthritis Index pain, baseline age, baseline body mass index, baseline Kellgren and Lawrence grade, baseline pain medication use, sex and race. Method 2. Odds of progression (radiographic and/or pain $(n=400)$ ) versus a non-progressor (progressor in neither radiographic nor pain $(n=200)$ ). Method 3. Odds of being a radiographic progressor $(n=297)$ versus radiographic non-progressor $(n=303)$. Method 4. Odds of being a pain progressor $(n=297)$ versus pain non-progressor $(n=303)$ for each 1 unit increase in biomarker.
The definition of radiographic progression relied upon change in the medial tibiofibular joint space. Perhaps not surprisingly, the odds for the MT and MF for area were generally larger than for the lateral tibiofemoral compartment and patella. Of greater interest, strength of association between bone biomarkers (area and shape) and progression appeared larger for the femur than for the tibia. This is consistent with other recent papers suggesting that femoral bone demonstrates greater change than tibial bone with regard to shape. ${ }^{16} 35$

Bone is a highly trophic and metabolically active tissue. In the context of OA, there are a number of alterations that occur in the periarticular bone. These include a progressive increase in subchondral plate thickness, alterations in the architecture of subchondral trabecular bone, formation of new bone at the joint margins (osteophytes), development of subchondral bone cysts and advancement of the tidemark associated with vascular invasion of the calcified cartilage. ${ }^{36}$ Other bone alterations in $\mathrm{OA}$ include bone marrow lesions, ${ }^{37}$ periostitis associated with osteophyte formation, ${ }^{38}$ subchondral microfractures, ${ }^{39}$ bone attrition $^{40}$ and bone ischaemia due to decreased blood flow and elevated intraosseous pressure. ${ }^{41}$

Broadly the alterations to subchondral bone architecture during the OA process are likely responsive to both adaptation to mechanical load and endochondral ossification. ${ }^{42}$ Both processes can lead to metaphyseal expansion and shape alteration that have been demonstrated as part of the OA pathophysio$\operatorname{logy}{ }^{43}$ As bone is principally responsible for load distribution in the weight-bearing knee, ${ }^{4}$ any kinematic change in loading is likely to lead to alteration in bone shape as it adapts to this changed load. Similarly, this responsive tissue may also demonstrate changes that are suggestive of deleterious progression towards an end stage osteoarthritic pathology. ${ }^{6}$ The reactivation of endochondral ossification and osteocartilaginous growth (osteophytes) may contribute to but does not explain all of the increased bone area. ${ }^{43}$ Previous studies have suggested that subtle alterations in joint shape at both the hip and knee may be involved in the pathogenesis of OA. ${ }^{9}{ }^{15}$ Prior studies using different methods of assessing bone area have demonstrated this 
construct to be able to predict the development of cartilage defects and the need for joint replacement, ${ }^{17}$ as well as be able to differentiate between different grades of severity of OA. ${ }^{14}$ This study adds to this literature and helps us to demonstrate the role bone area and shape play in predicting clinically relevant disease progression.

Several limitations of this work warrant recognition. We modelled the shape change of whole bones, but local regional changes may be more sensitive. There are a number of important factors that likely contribute to bone area and shape that we have adjusted for including JSW, age, BMI, KLG, sex and race; however, other factors were not accounted for such as use of bisphosphonates and physical activity levels. The main results present the longitudinal validity of these bone biomarkers; however, there is the potential for some of this being concurrent as opposed to predictive validity as case status could be defined from 24 to 48 (ie, after the 12-month visit) months with our independent variable being assessed from baseline to 24 months. The preliminary analyses we have conducted from baseline to 12 months, however, strengthen the inference for predictive as opposed to concurrent validity. The case-control definition focuses on persons with medial tibiofemoral OA, many of whom may have disease in other compartments of the knee including the patellofemoral compartment that could account for some of their symptoms.

Unfortunately at present there is no OA equivalent to measuring glycosylated haemoglobin (HbA1c) as we have for diabetes, where one can monitor and treat the disease precursors preemptively before the associated processes lead to end-organ failure. ${ }^{44}$ In addition in OA, even if we had such a biomarker, there are no therapies proven to reduce the risk of progression to OA. ${ }^{45}$ Instead, the 'watchful waiting' of steady decline to end-stage joint disease is a major cause of disablement and loss of quality of life. ${ }^{46}$ While the reasons for the translational failure of disease-modifying OA drugs (DMOADs) are likely multifold, they include the absence of responsive biomarkers that predict clinically relevant worsening of structure and symptoms in knee OA for use in clinical trials. Another critical challenge and obstacle to further therapeutic development is the heterogeneity of OA. At present, there are marked limitations with the way we define and classify subgroups of $\mathrm{OA}$ and it may be that certain biomarkers performed better in particular phenotypes than others. At this point it remains unclear whether there is a specific phenotype within the heterogeneous disease of $\mathrm{OA}$ that the bone shape and area biomarker will be best applied within, and it is important to note that this sample focuses on those with medial tibiofemoral OA.

Despite the limitations of this study, this work demonstrates that these imaging biomarkers have promise in predicting clinically meaningful progression in persons with knee OA. Further work is required before this will immediately address the complex reasons for failure of DMOAD trials and regulatory approval of DMOADs. There are many reasons for the translational failure of anti-OA drugs, including the poor relationship in individual patients between joint structural pathology (especially JSN on radiographs) and symptomatic disease, the heterogeneity of disease and limited responsiveness of existing biomarkers. ${ }^{44}$ This study is one solid step in the right direction towards improving biomarker performance and validation. Further work is required on the responsiveness of these measures, how they compare with other MRI and biochemical measures, whether they may have baseline prognostic utility in predicting those at risk of more rapid progression, as well as their performance as an efficacy of intervention marker in clinical trials. ${ }^{47}$ Irrespective, these results demonstrate that alteration in bone area and shape over a 2-year period have significant associations with disease progression over 4 years, providing a compelling rationale for greater research focus on these parameters for therapeutic target development and disease modification.

\section{Author affiliations}

${ }^{1}$ Rheumatology Department, Royal North Shore Hospital and Institute of Bone and Joint Research, Kolling Institute, University of Sydney, Sydney, New South Wales, Australia

${ }^{2}$ Department of Epidemiology and Biostatistics, University of California San Francisco, San Francisco, California, USA

${ }^{3}$ Duke Molecular Physiology Institute and Division of Rheumatology, Duke University School of Medicine, Durham, North Carolina, USA

${ }^{4}$ Brigham and Women's Hospital, Boston, Massachusetts, USA

${ }^{5}$ Imorphics, Manchester, UK

${ }^{6}$ Department of Radiology, Boston University School of Medicine, Quantitative Imaging Center, Boston, Massachusetts, USA

${ }^{7}$ Department of Radiology, University of Erlangen-Nuremberg, Erlangen, Germany

\section{Twitter Follow David Hunter at @ProfDavidHunter}

Acknowledgements We thank the Osteoarthritis Research Society International (OARSI) for their leadership and expertise on the FNIH project.

Contributors Study conception and design: DH, JEC, MN, VBK, JNK and EL. Acquisition of data: $M B, J E C, M N$ and JL. Analysis and interpretation of data: $\mathrm{DH}$, JEC, MN, VBK, JNK, EL, FWR, AG and MB. Writing of first manuscript draft: DH. Critical manuscript revision and approval of final manuscript: all authors. DH had full access to all of the data in the study and takes responsibility for the integrity of the data and the accuracy of the data analysis.

Funding Scientific and financial support for the FNIH OA Biomarkers Consortium and the study are made possible through grants and direct contributions provided by AbbVie; Amgen; Arthritis Foundation; Bioiberica S.A; DePuy Mitek; Flexion

Therapeutics; GlaxoSmithKline; Merck Serono; Rottapharm Madaus; Sanofi; and Stryker; The Pivotal OAI MRI Analyses (POMA) Study, NIH HHSN2682010000. The OAl is a public-private partnership comprised of five contracts (N01-AR-2-2258; N01-AR-2-2259; N01-AR-2-2260; N01-AR-2-2261; N01-AR-2-2262) funded by the National Institutes of Health. Funding partners include Merck Research Laboratories; Novartis Pharmaceuticals Corporation, GlaxoSmithKline; and Pfizer. Private sector funding for the Consortium and OAl is managed by the FNIH.

Competing interests DH is funded by an NHMRC Practitioner Fellowship and is supported from the FNIH OA Biomarkers Consortium. VK, MN, JC and EL are funded by grants from the FNIH. EL is funded by grants from NIAMS R01 AR064320, K24 AR057827, P60 AR47782. MB is a part-time employee and co-owner of Imorphics. AG is President of Boston Imaging Core Lab, LLC (BICL), a company providing MRI reading services to academic researchers and to industry. He has provided consulting services to Merck Serono, Genzyme, OrthoTrophix and TissueGene. FWR is CMO and co-owner of BICL.

\section{Patient consent Obtained.}

Ethics approval The OAI study received ethical approval from each OAI clinical site and for the OAl coordinating center. All participants provided informed consent to the OAl.

Provenance and peer review Not commissioned; externally peer reviewed.

\section{REFERENCES}

1 Hunter DJ, Schofield D, Callander E. The individual and socioeconomic impact of osteoarthritis. Nat Rev Rheumatol 2014;10:437-41.

2 Vos T, Flaxman AD, Naghavi M, et al. Years lived with disability (YLDs) for 1160 sequelae of 289 diseases and injuries 1990-2010: a systematic analysis for the Global Burden of Disease Study 2010. Lancet 2012;380:2163-96.

3 Losina E, Walensky RP, Reichmann WM, et al. Impact of obesity and knee osteoarthritis on morbidity and mortality in older americans. Ann Intern Med 2011;154:217-26.

4 Hoshino A, Wallace WA. Impact-absorbing properties of the human knee. J Bone Joint Surg Br 1987;69:807-11.

5 van Spil WE, DeGroot J, Lems WF, et al. Serum and urinary biochemical markers for knee and hip-osteoarthritis: a systematic review applying the consensus BIPED criteria. Osteoarthritis Cartilage 2010;18:605-12.

6 Kraus VB, Feng $S$, Wang $S$, et al. Trabecular morphometry by fractal signature analysis is a novel marker of osteoarthritis progression. Arthritis Rheum 2009;60:3711-22.

7 Messent EA, Ward RJ, Tonkin CJ, et al. Tibial cancellous bone changes in patients with knee osteoarthritis. A short-term longitudinal study using Fractal Signature Analysis. Osteoarthritis Cartilage 2005;13:463-70. 
8 Kraus VB, Feng $S$, Wang $S$, et al. Subchondral bone trabecular integrity predicts and changes concurrently with radiographic and magnetic resonance imagingdetermined knee osteoarthritis progression. Arthritis Rheum 2013;65:1812-21.

9 Haverkamp DJ, Schiphof D, Bierma-Zeinstra SM, et al. Variation in joint shape of osteoarthritic knees. Arthritis Rheum 2011;63:3401-7.

10 Bredbenner TL, Eliason TD, Potter RS, et al. Statistical shape modeling describes variation in tibia and femur surface geometry between Control and Incidence groups from the osteoarthritis initiative database. J Biomech 2010:43:1780-6.

11 Grynpas MD, Alpert B, Katz I, et al. Subchondral bone in osteoarthritis. Calcif Tissue Int 1991;49:20-6.

12 Radin EL, Rose RM. Role of subchondral bone in the initiation and progression of cartilage damage. Clin Orthop Relat Res 1986;213:34-40.

13 Barr AJ, Dube B, Hensor EM, et al. The relationship between clinical characteristics, radiographic osteoarthritis and $3 \mathrm{D}$ bone area: data from the Osteoarthritis Initiative. Osteoarthritis Cartilage 2014;22:1703-9.

14 Frobell RB, Nevitt MC, Hudelmaier M, et al. Femorotibial subchondral bone area and regional cartilage thickness: a cross-sectional description in healthy reference cases and various radiographic stages of osteoarthritis in 1,003 knees from the Osteoarthritis Initiative. Arthritis Care Res (Hoboken) 2010;62: 1612-23.

15 Ganz R, Parvizi J, Beck M, et al. Femoroacetabular impingement: a cause for osteoarthritis of the hip Clin Orthop Relat Res 2003;417:112-20.

16 Neogi T, Bowes MA, Niu J, et al. Magnetic resonance imaging-based three-dimensional bone shape of the knee predicts onset of knee osteoarthritis: data from the osteoarthritis initiative. Arthritis Rheum 2013;65:2048-58.

17 Ding C, Cicuttini F, Jones G. Tibial subchondral bone size and knee cartilage defects: relevance to knee osteoarthritis. Osteoarthritis Cartilage 2007;15: 479-86.

18 Hunter DJ, Nevitt M, Losina E, et al. Biomarkers for osteoarthritis: current position and steps towards further validation. Best Pract Res Clin Rheumatol 2014;28:61-71.

19 Lester G. Clinical research in OA-the NIH Osteoarthritis Initiative. J Musculoskelet Neuronal Interact 2008:8:313-14.

20 Peterfy CG, Schneider E, Nevitt M. The osteoarthritis initiative: report on the design rationale for the magnetic resonance imaging protocol for the knee. Osteoarthritis Cartilage 2008;16:1433-41.

21 Felson D, Niu J, Sack B, et al. Progression of osteoarthritis as a state of inertia. Ann Rheum Dis 2013;72:924-9.

22 Peterfy C, Li J, Zaim S, et al. Comparison of fixed-flexion positioning with fluoroscopic semi-flexed positioning for quantifying radiographic joint-space width in the knee: test-retest reproducibility. Skeletal Radiol 2003;32:128-32.

23 Felson DT, Nevitt MC, Yang M, et al. A new approach yields high rates of radiographic progression in knee osteoarthritis. J Rheumatol 2008;35: 2047-54.

24 Felson DT, Niu J, Guermazi A, et al. Defining radiographic incidence and progression of knee osteoarthritis: suggested modifications of the Kellgren and Lawrence scale. Ann Rheum Dis 2011;70:1884-6.

25 Kellgren JH, Lawrence JS. Atlas of standard radiographs. Oxford: Blackwell Scientific, 1963.

26 Altman RD, Gold GE. Atlas of individual radiographic features in osteoarthritis, revised. Osteoarthritis Cartilage 2007;15(Suppl A):A1-56.

27 Wirth W, Duryea J, Hellio Le Graverand MP, et al. Direct comparison of fixed flexion, radiography and MRI in knee osteoarthritis: responsiveness data from the Osteoarthritis Initiative. Osteoarthritis Cartilage 2013;21:117-25.
28 Altman RD, Hochberg M, Murphy WAJ, et al. Atlas of individual radiographic features in osteoarthritis. Osteoarthritis Cartilage 1995;3(Suppl A):3-70.

29 Bellamy N, Buchanan WW, Goldsmith $\mathrm{CH}$, et al. Validation study of WOMAC a health status instrument for measuring clinically important patient relevant outcomes to antirheumatic drug therapy in patients with osteoarthritis of the hip or knee. J Rheumatol 1988;15:1833-40.

30 Angst F, Aeschlimann A, Stucki G. Smallest detectable and minimal clinically important differences of rehabilitation intervention with their implications for required sample sizes using WOMAC and SF-36 quality of life measurement instruments in patients with osteoarthritis of the lower extremities. Arthritis Rheum 2001;45:384-91

31 Angst F, Aeschlimann A, Michel BA, et al. Minimal clinically important rehabilitation effects in patients with osteoarthritis of the lower extremities. J Rheumatol 2002;29:131-8

32 Bowes MA, Vincent GR, Wolstenholme CB, et al. A novel method for bone area measurement provides new insights into osteoarthritis and its progression. Ann Rheum Dis 2015;74:519-25.

33 Hunter DJ, Bowes MA, Eaton CB, et al. Can cartilage loss be detected in knee osteoarthritis $(\mathrm{OA})$ patients with 3-6 months' observation using advanced image analysis of 3T MRI? Osteoarthritis Cartilage 2010;18:677-83.

34 Eckstein F, Ateshian G, Burgkart R, et al. Proposal for a nomenclature for magnetic resonance imaging based measures of articular cartilage in osteoarthritis. Osteoarthritis Cartilage 2006;14:974-83.

35 Hunter DJ, Lohmander LS, Makovey J, et al. The effect of anterior cruciate ligament injury on bone curvature: exploratory analysis in the KANON trial. Osteoarthritis Cartilage 2014;22:959-68.

36 Hunter DJ, Guermazi A, Roemer F, et al. Structural correlates of pain in joints with osteoarthritis. Osteoarthritis Cartilage 2013;21:1170-8.

37 Roemer FW, Frobell R, Hunter DJ, et al. MRI-detected subchondral bone marrow signal alterations of the knee joint: terminology, imaging appearance, relevance and radiological differential diagnosis. Osteoarthritis Cartilage 2009;17:1115-31.

38 Cicuttini FM, Baker J, Hart DJ, et al. Association of pain with radiological changes in different compartments and views of the knee joint. Osteoarthritis Cartilage 1996:4:143-7.

39 Burr DB. The importance of subchondral bone in the progression of osteoarthritis. J Rheumatol Supp/ 2004;70:77-80.

40 Torres L, Dunlop DD, Peterfy C, et al. The relationship between specific tissue lesions and pain severity in persons with knee osteoarthritis. Osteoarthritis Cartilage 2006;14:1033-40.

41 Simkin P. Bone pain and pressure in osteoarthritic joints. Novartis Found Symp 2004;260:179-86.

42 Cox LG, van Donkelaar CC, van Rietbergen B, et al. Alterations to the subchondral bone architecture during osteoarthritis: bone adaptation vs endochondral bone formation. Osteoarthritis Cartilage 2013;21:331-8.

43 Brandt KD, Dieppe P, Radin EL. Etiopathogenesis of osteoarthritis. Rheum Dis Clin North Am 2008;34:531-59.

44 Hunter DJ, Losina E, Guermazi A, et al. A pathway and approach to biomarker validation and qualification for osteoarthritis clinical trials. Curr Drug Targets 2010;11:536-45

45 Matthews GL, Hunter DJ. Emerging drugs for osteoarthritis. Expert Opin Emerg Drugs 2011;16:479-91.

46 Hunter DJ. Osteoarthritis. Best Pract Res Clin Rheumatol 2011;25:801-14.

47 Bauer DC, Hunter DJ, Abramson SB, et al. Classification of osteoarthritis biomarkers: a proposed approach. Osteoarthritis Cartilage 2006;14:723-7. 